\title{
Adaptive responses of independent traits to the same environmental gradient in the intertidal snail Bembicium vittatum
}

\author{
MS Johnson and R Black \\ School of Animal Biology (M092), University of Western Australia, Crawley, WA, Australia
}

\begin{abstract}
The snail Bembicium vittatum occupies a wide range of intertidal habitats in the Houtman Abrolhos Islands, Western Australia. Allozyme variation reflects patterns of connectivity, which are independent of local habitat. In contrast, heritable differences in shell shape among 83 shore sites vary with habitat, indicating local adaptation. Here we examine dimorphisms of colour and spotting of the shell in the same populations, as a test of consistency and complexity of patterns of local adaptation. Within populations, the frequency of spotted shells is higher in dark shells. Despite this association, spatial variations of colour and spotting are only weakly correlated. As predicted for traits associated with local adaptation, subdivision is greater for colour, spotting and shape than for allozymes. Colour and shape are associated with local habitat, such that populations on vertical shores have higher frequencies of dark and relatively
\end{abstract}

Keywords: adaptation; shell morph; genetic subdivision; gastropod; $F_{\mathrm{ST}} ; Q_{\mathrm{ST}}$

\section{Introduction}

Understanding genetic structure of populations is fundamental to the study of evolutionary change and to basic issues in conservation genetics. Genetic structure is usually examined using molecular markers, such as allozymes, mtDNA sequences, microsatellite DNA and amplified fragment length polymorphisms (AFLPs; Avise, 2004). Such markers typically behave as selectively neutral or at least quasi-neutral, and hence are useful for inferring patterns of gene flow and historical connections among populations, as well as for quantifying genetic variation within populations. Such molecular markers provide a structural and historical context in which adaptive evolution might occur, but their relationship to adaptive change is unclear, because these markers are generally poor indicators of functionally significant traits (for example, Lynch, 1996; Pearman, 2001; Reed and Frankham, 2001). A high degree of genetic subdivision indicates the opportunity for local adaptation, but isolation also increases the potential for random drift to dominate. In contrast to neutral molecular markers,

Correspondence: Professor MS Johnson, School of Animal Biology (M092), University of Western Australia, 35 Stirling Highway, Crawley, WA 6009, Australia.

E-mail:msj@cyllene.uwa.edu.au

Received 15 November 2007; revised 18 March 2008; accepted 24 March 2008; published online 7 May 2008 flatter shells than those on gently sloping shores. These associations are repeatable between three separate groups of islands. Spotting shows a weaker, but significant association with the same gradient. Although shape does not differ between colour morphs within populations, the proportion of dark shells is strongly associated with shape. Thus, the independent shell traits are apparently adapted to a common, biologically significant gradient, even though the adaptive mechanisms probably differ for colour and shape. The parallel variations of independent traits highlight both the complexity of local adaptation and the potential to reveal evolutionarily significant environmental contrasts by examining adaptively relevant traits.

Heredity (2008) 101, 83-91; doi:10.1038/hdy.2008.33; published online 7 May 2008 phenotypic traits are more likely to reflect adaptive variation, and such traits often show different patterns of divergence to those of molecular markers (Lynch, 1996; McKay and Latta, 2002; van Tienderen et al., 2002).

Although not directly relevant to adaptive divergence, neutral molecular markers provide a valuable framework for detecting adaptively significant variation. For example, associations of traits with habitat are potential evidence of local adaptation, but confounding effects of history or gene flow must be taken into account. Neutral markers can reveal whether associations of phenotypic traits with habitat are independent of patterns of gene flow or historical connections, and therefore whether the associations provide strong evidence for local adaptation (Schneider et al., 1999; Quesada et al., 2007). A related use of neutral markers for recognition of significant traits is based on the prediction that adaptively relevant traits should show greater subdivision than neutral markers. This notion was clearly expressed by Lewontin and Krakauer (1973) as a test for natural selection, in the context of identifying disparities among allozyme loci. As expected from this perspective, levels of genetic subdivision for phenotypic traits are often higher than those for molecular markers (Spitze, 1993; Gockel et al., 2001; Storz, 2002; Steinger et al., 2002; Luttikhuizen et al., 2003; Volis et al., 2005; Schiffer et al., 2007). This approach has also been the basis for detecting subsets of potentially important loci, when using AFLP markers as genomic scans (Beaumont, 2005; Storz, 2005), with 
important implications for using genetics to set priorities in conservation (Bonin et al., 2007).

Intertidal snails provide useful opportunities for testing contrasting patterns of genetic divergence for molecular markers and potentially adaptive morphological variation, because they are readily accessible and often occupy highly contrasting habitats (reviews in McQuaid, 1996; Reid, 1996). An example is the littorine Bembicium vittatum in the Houtman Abrolhos Islands, an archipelago of more than 100 small islands, $70 \mathrm{~km}$ off the Western Australian coast. This species lacks planktonic larvae, and consequently is genetically subdivided over distances of tens to hundreds of metres (Johnson and Black, 1995, 1996, 1998a). The species is abundant in the Abrolhos Islands, where it occupies a wide range of habitats. The advantages of B. vittatum in the Abrolhos Islands for studying adaptive genetic divergence are threefold: (1) analysis of many, well-characterized sites provides statistical power and well-defined environmental context (Johnson and Black, 1996, 1997); (2) analysis of molecular variation has shown clear patterns of genetic connectivity, but these are independent of any measured features of the local intertidal habitat, indicating that associations of other traits with habitat are not due to confounding effects of gene flow (Johnson and Black, 1995, 1996, 2006); (3) replication of habitat characteristics among three (genetically divergent) island groups allows rigorous testing of associations with habitat (Johnson and Black, 1996, 2000). Habitats occupied by B. vittatum differ in substrate, slope of the shore, exposure to currents and wind, and associated biological communities. These contrasting habitats provide a specific context in which local, adaptive divergence can be studied.

In common with many intertidal snails, B. vittatum is variable for shell shape and pigmentation. Variations in shape and spotting of the shell are inherited (Parsons, 1997), and hence are candidates for local adaptation. The functional importance of such traits is understood in broad terms for intertidal snails generally and for littorines in particular (Vermeij, 1993; McQuaid, 1996; Reid, 1996). In B. vittatum, there are close associations between shell shape and local habitat, which are consistent among the three island groups and are independent of patterns of allozyme variation, indicating that these populations are locally adapted (Johnson and Black, 2000).

In the present study, we examine variation in shell colour and spotting in B. vittatum among the same populations studied previously for allozymes and shell shape. We compare levels of subdivision and distribution of variation within and between island groups for the individual shell traits and allozymes, as indirect indicators of traits that might be locally adapted. We then test each trait for associations with local habitat, to determine which traits contribute to identifiable local adaptation, and whether the adaptive traits vary together on the same environmental gradients.

\section{Methods}

Sample sites and habitat

Samples of adult B. vittatum were collected at 83 shore sites from throughout the Houtman Abrolhos Islands. These samples were analysed previously for variation in size and shape (Johnson and Black, 2000), and all but two of them were used in the allozyme study of genetic divergence (Johnson and Black, 1996). The average sample size was 52, and only four sites had fewer than 33 snails $(N=17-26)$. The samples represent the species' distribution throughout the Abrolhos Islands, spanning nearly $80 \mathrm{~km}$, and include the major habitats in all three of the separate island groups

Although contrasts between high and low on the shore are important for local adaptation of some intertidal species (Grahame et al., 2006; Quesada et al., 2007), $B$. vittatum in the Abrolhos Islands occupies a tidal range of $<0.5 \mathrm{~m}$, and environmental heterogeneity is modest within sites. In contrast, there are substantial differences of habitat among sites occupied by B. vittatum. Quantitative descriptions of the habitats at 71 of the sites were made previously, based on 31 variables that include indicators of exposure, water flow, substrate, shore profile and biological communities (Johnson and Black, 1996, 1997). These variables provide a clear description of the major variations in habitat, and are good predictors of the distributions of high intertidal gastropods in the Abrolhos Islands (Johnson and Black, 1997). To provide composite indices, the habitat variables were subjected to principal components analysis, calculated using the Statview SE + Graphics program (Abacus Concepts Inc.). The raw principal components were used, providing orthogonal (statistically independent) axes of variation in habitat. The first three principal components are readily interpretable descriptors: PC1 represents exposure to wave action and water flow; PC2 the gradient between vertical and sloping shores; PC3 aspect in relation to prevailing winds (Johnson and Black, 1996).

\section{Shell polymorphism}

Shells were scored for the presence of dark spots on the ventral surface and for colour. Spotting is inherited, with the spotted condition being dominant to unspotted (Parsons, 1997). The number of spots varies, but any shell with at least two spots was classified as spotted. In addition to variation in spotting, there is a dimorphism in ground colour, with shells being either pale or dark (Figure 1). Although occasional shells have some discontinuities in the dark pigment, their frequency is less than $2 \%$ in our samples, and any shell with dark pigment in the interior lining of the shell was classified as dark.

Shape of the shell also varies substantially, from relatively tall, beehive shape to relatively flat, and this

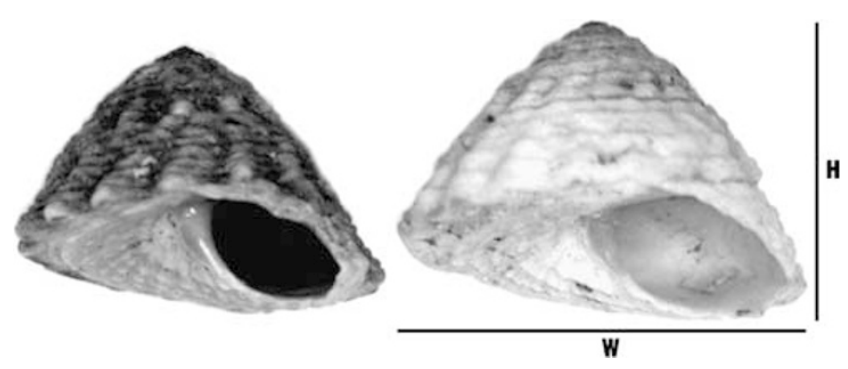

Figure 1 Dark- (left) and pale-colour morphs of Bembicium vittatum. Measurements of shell width $(W)$ and height $(H)$ are indicated for the pale shell. 
variation of relative shell height among populations is highly heritable (Parsons, 1997). Shell shape varies with habitat (Johnson and Black, 2000) and was included in the analysis of geographic variation of shell colour and spotting, as an indicator of habitat variation. Shells from the populations included here were measured for width (maximum diameter, perpendicular to the columella; Figure 1) and height (maximum distance parallel to the columella; Figure 1) in the previous study. Shell shape was expressed as relative height, whereby height was standardized by regression within each population to a standard width of $8.5 \mathrm{~mm}$ (Johnson and Black, 2000). This relative (to the standard shell width) height provides a measure of shape that is independent of shell size, and its average value within populations varies from 3.9 (relatively flat shells) to $7.2 \mathrm{~mm}$ (tall shells). Relative height was used in the present study, for comparisons with spotting and colour, both within and between populations.

To determine whether the phenotypic traits were independent, associations were tested within populations. Association of spotting and colour within each population was tested by a $2 \times 2$ contingency test. Mean relative shell heights were compared between morphs by $t$-tests within each population, and paired $t$-tests were used to test for an overall trend of difference in shape between the morphs across all the populations.

\section{Analyses of geographic variation}

Variation of allozymes among these sites follows patterns of connectivity, but is not associated with local habitat (Johnson and Black, 1996). Variation of the shell traits was compared with that for allozymes in two ways, as possible indications of adaptive variation. First, because traits involved in local adaptation are likely to show higher levels of subdivision, subdivision across all populations was quantified as $F_{\mathrm{ST}}$ for colour and spotting, and as the corresponding $Q_{\mathrm{ST}}$ (Spitze, 1993) for relative shell height, and compared with the value for allozymes from Johnson and Black (1996). To calculate $F_{\mathrm{ST}}$ for the shell morphs, we estimated allelic frequencies, based on the assumptions that variation of colour and spotting is determined by pairs of alleles at single loci, with dark and spotted being dominant, and that each locus is in Hardy-Weinberg equilibrium. Experimental matings support the assumed simple Mendelian inheritance of spotting (Parsons, 1997); the assumption that the same applies to colour is based on the distinct dimorphism and the association of colour and spotting within populations (details in 'Results').

For relative shell height, $Q_{\mathrm{ST}}$ was estimated as the between-site variance, as a proportion of the sum of the within- and between-site variances. Although this measure is based on phenotypic variance, it is likely to be very close to the to the true $Q_{\mathrm{ST}}$. Matings among three source populations confirmed that differences in shell shape among populations are highly heritable, with an $r^{2}$ of 0.95 between parental (native populations) and offspring (experimental matings) (Parsons 1997). Furthermore, phenotypic variance may actually underestimate genotypic variance: slower growth produces slightly taller shells for a given width, but growth is slower (that is, tending to produce relatively taller shells) at the vertical shores, where shells are relatively flat
(Johnson and Black, 1998b). This countergradient variation strengthens the view that we have not materially overestimated $Q_{\mathrm{ST}}$. Standard errors for $Q_{\mathrm{ST}}$ and $F_{\mathrm{ST}}$ for the shell traits were estimated from 1000 psuedoreplicates from bootstrapping across populations, using Resampling Statistics (Simon, 1995).

The second comparison with allozymes was a partition of genetic subdivision between and within the three island groups. Intertidal habitats vary within groups, and do not differ between the groups (Johnson and Black, 1997), but nearly half the geographic variation of allozymes is between groups (Johnson and Black, 1996). Thus, partitioning of variation of the shell traits provides a test of whether they reflect the pattern of isolation indicated by the allozymes. North Island (Figure 2) was included in the Wallabi group for this analysis. For colour and spotting, partitioning followed Nei's method, using the estimated allelic frequencies (Nei, 1973). For relative shell height, the total variance among populations was partitioned by restricted maximum likelihood into variance components within and between island groups, using JMP version 5.1 (SAS Institute). Variance components were expressed as percentages of the total variance among populations. Differences of morph frequencies and relative shell height among the three island groups were also tested by single-factor analysis of variance, in Statview SE + Graphics.

As a more direct search for local adaptation, regression analysis was used to test for geographic associations of the proportions of spotted or dark shells with (1) habitat PCs 1-3 and (2) relative shell height. The regressions of morph frequency on habitat variables and shell height were done over the entire set of sites (71 for habitat PCs, 83 for relative shell height) and within each island group separately ( $n=17$ for the Wallabi group, 24 or 28 for the Easter group, and 30 or 38 for the Pelsaert group), to test for robustness of any associations with habitat.

\section{Results}

The frequencies of the colour and spotting morphs in the 83 populations are mapped in Figure 2, which shows three basic features. First, there was substantial variation in frequencies of both traits: the proportion of dark shells ranged from 0.01 to 1.00 , and that of spotting from 0 to 0.87 . Second, variation was local, rather than between the island groups, as indicated by large differences in frequencies within each group.

The third feature evident in Figure 2 is an association between colour and spotting, indicated most clearly by the relative scarcity of pale shells with spots. Spotting was more common in dark shells than in pale shells at all but 2 of the 70 sites where both colour and spotting were polymorphic (Figure 3). The association ranged from moderate to complete, and was statistically significant $(P<0.05)$ at 43 sites. At the two exceptional sites, where spots were more common in pale shells, the association was weak and non-significant (Figure 3).

As expected from the association of traits within populations, the proportions of spotted shells and of dark shells were associated among all 83 sites, as high proportions of spotted shells occurred only at sites with high proportions of dark shells (Figure 4). Nevertheless, the geographic association was weak, indicating substantial independence in the spatial variation of colour 


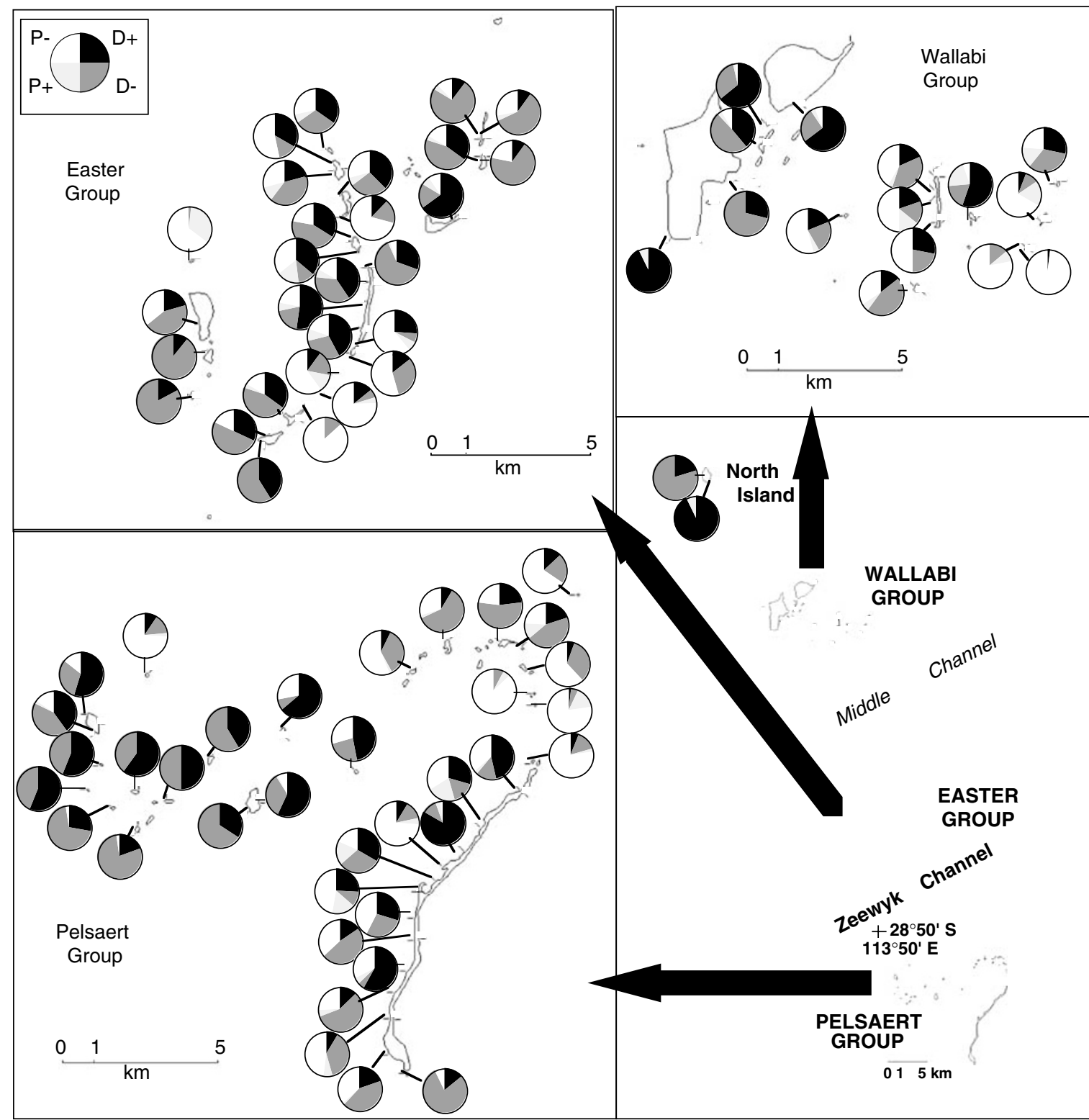

Figure 2 Proportions of spotting and colour morphs in samples of Bembicium vittatum from the Abrolhos Islands. P, pale; D, dark; + , spotted; - , unspotted.

and spotting: nearly the full range of frequencies of dark shells occurred among populations with few spotted shells, and nearly the full range of frequencies of spotted shells occurred among populations with high frequencies of dark shells. These patterns applied across all three groups of islands (Figure 4), highlighting again that variation of morph frequencies was local, rather than between groups.

Both the amount of subdivision and its partitioning differed between the shell traits and the allozymes (Table 1). The estimates of $F_{\mathrm{ST}}$ were 0.585 for colour and 0.358 for spotting, and $Q_{\mathrm{ST}}$ was 0.557 for relative shell height, each value being several standard errors above the mean $F_{\mathrm{ST}}$ of 0.206 across all 14 allozyme loci, and outside the range of values for the individual allozyme loci. Furthermore, less than $2 \%$ of the divergence of the individual shell traits was between the three island groups, compared to $46 \%$ for the allozymes
(Table 1). The lack of differentiation between groups was confirmed by single-factor analyses of variance, which did not detect differences between the three island groups for the frequencies of colour $\left(F_{2,80}=0.083\right.$, $P>0.90)$ or spotting $\left(\mathrm{F}_{2,80}=0.342, P>0.70\right)$, or for mean relative shell height $\left(\mathrm{F}_{2,80}=1.059, P>0.35\right)$.

Rather than being associated with island groups, morph frequencies varied with habitat. Frequencies were tested for association with habitat, using regressions on the first three habitat principal components, and the clearest patterns were for shell colour. Among the 71 sites for which habitat characteristics were measured, there was a negative association of the proportion of dark shells with habitat PC2, which represents a gradient of vertical to sloping shores $\left(r^{2}=0.319, P<0.0001\right)$. Importantly, this association was consistent within each of the three island groups (Figure 5, upper); the regressions were significant within each group, and neither the 


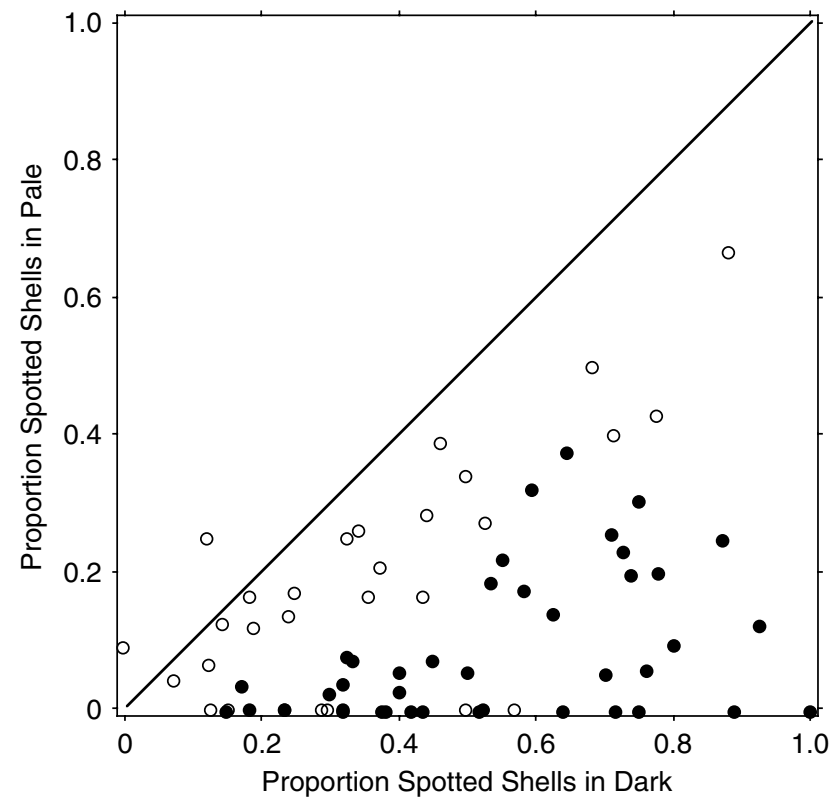

Figure 3 Comparison of frequencies of spotted shells within pale and dark morphs, in each of 70 populations polymorphic for both traits. Diagonal indicates equal proportions of spotted morph in pale and dark shells. Filled circles indicate significant $(P<0.05)$ association of spotting with dark shells.

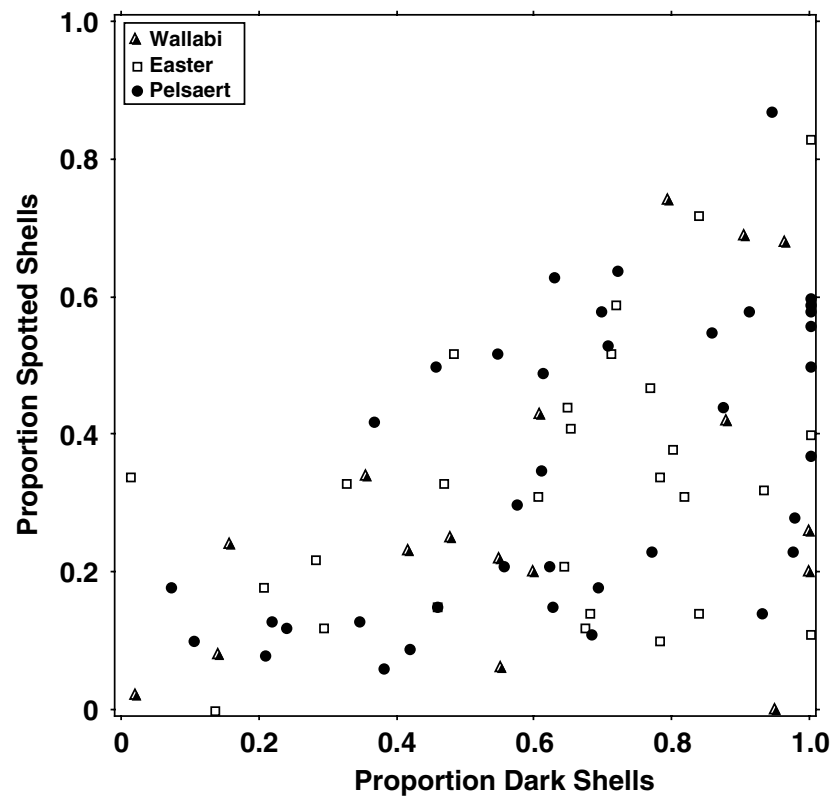

Figure 4 Association of geographic variation of the proportions of spotted shells and dark shells among 83 populations, coded by island group. slopes nor the elevations of the regressions differed between island groups.

The association of dark shells with habitat paralleled that previously found for relative shell height (Johnson and Black, 2000), as both dark shells and relatively flat shells were associated with vertical shores. The proportion of dark shells was strongly associated with relative shell height $\left(r^{2}=0.397, P<0.0001\right.$; Figure 5, lower). This association with relative shell height was also significant within each of the three island groups, and the slopes and elevations of the regressions did not differ between groups, confirming the repeatability of the relationship.

The parallel geographic variation of colour and shape was not due to intrinsic association of the two traits. We tested for independence of colour and shell shape by comparing average relative shell height between dark and pale shells within each population represented by at least 10 snails of each morph (44 sites). Only one site showed a significant $(P=0.031)$ difference in shape between dark and pale shells, but this significance disappeared when correction was made for multiple tests. There was no overall tendency towards a difference in relative shell height between dark and pale shells (paired $t$-test, $t=0.932, P>0.35$ )

Despite its consistent association with colour within populations, spotting was only weakly associated with PC2 $\left(r^{2}=0.123, P=0.0027\right)$ and relative shell height $\left(r^{2}=0.066, P=0.0019\right)$, and was not associated with the other habitat axes. To control for the effect of its association with colour, the proportions of spotted shells within only the dark shells or within only the pale shells were tested. Because these analyses were based on smaller samples, we restricted them to sites with at least 30 individuals of the particular colour morph (52 sites for dark shells, 17 for pale shells). The small, independent association of spotting in dark shells with habitat PC2 was significant $\left(r^{2}=0.100, P=0.036\right)$, but the weaker association in the weaker test for association in pale shells was not $\left(r^{2}=0.048, P=0.396\right)$. Independent support for the association of spotting in dark shells with habitat was found in the Pelsaert group (19 sites, $\left.r^{2}=0.260, P=0.026\right)$ and the Easter group (16 sites, $\left.r^{2}=0.245, P=0.051\right)$, indicating a modest, but significant association of spotting with habitat, independent of its association with colour. When colour morph was held constant, none of the regressions of frequencies of spotted shells with relative shell height were significant, further highlighting that colour and spotting differ in their patterns of geographic variation.

\section{Discussion}

The main findings of this study are the consistent association of colour and spotting within populations,

Table 1 Levels of subdivision $\left(F_{\mathrm{ST}}\right.$ for allozymes, colour and spotting; $Q_{\mathrm{ST}}$ for shell height $) \pm$ s.e. among 83 populations of $B$. vittatum, and partitions of inter-population variation within and between island groups

\begin{tabular}{lcccc}
\hline & Allozymes & Colour & Spotting & Shell height \\
\hline Subdivision & $0.206 \pm 0.023(0.022-0.338)$ & $0.585 \pm 0.034$ & $0.358 \pm 0.026$ & $0.557 \pm 0.039$ \\
& & & & \\
Proportion variance & 0.540 & 0.997 & 0.983 & 0.985 \\
Within-island groups & 0.460 & 0.003 & 0.017 & 0.015 \\
Between-island groups & & & \\
\hline
\end{tabular}

For the allozymes, range of $F_{\mathrm{ST}}$ of 14 polymorphic loci is given in parentheses. 


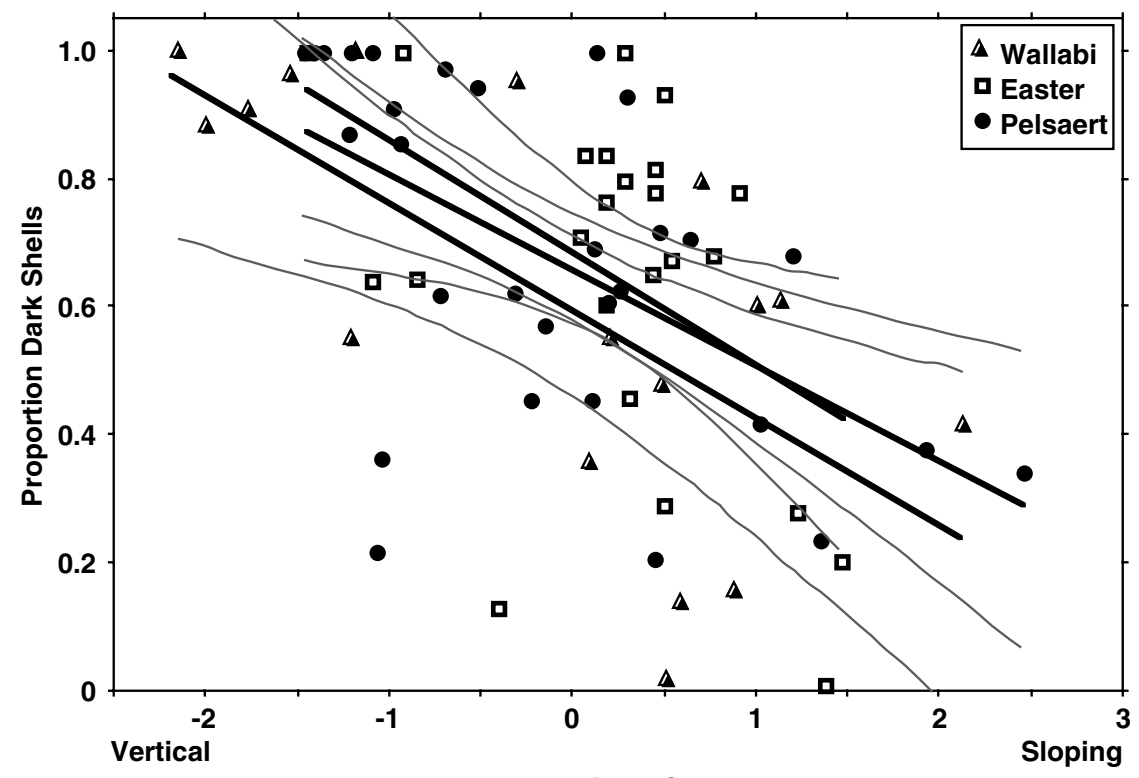

Habitat PC2

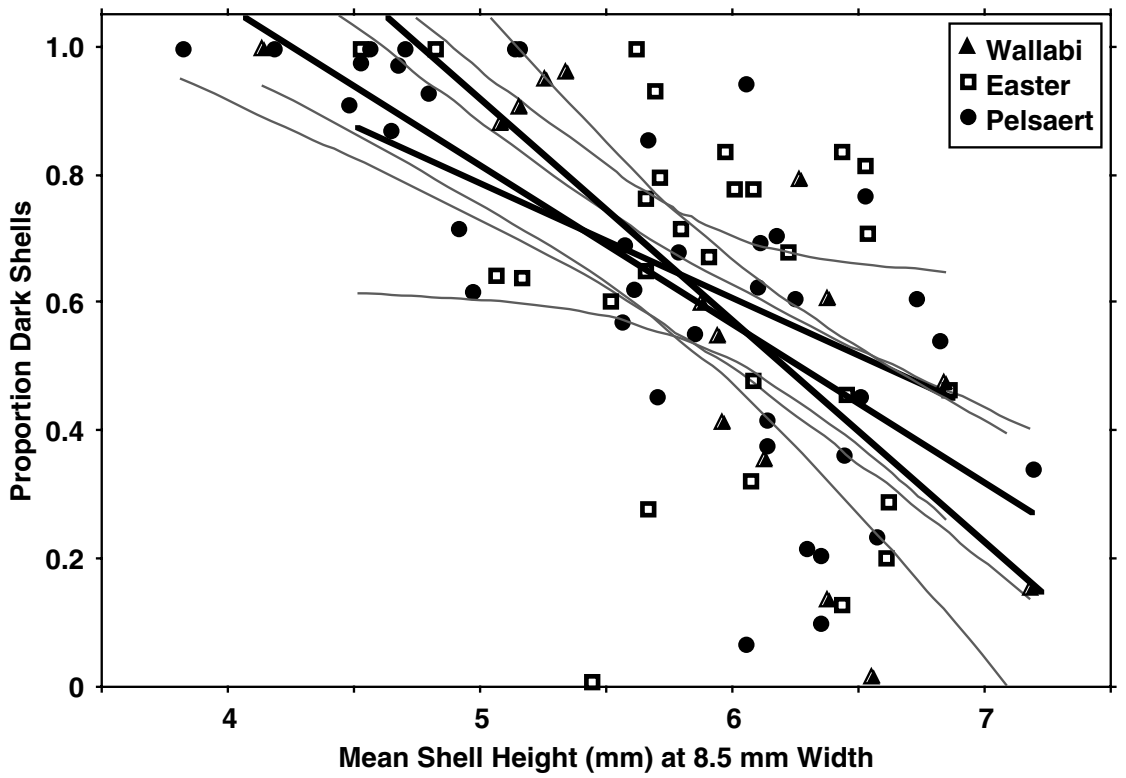

Figure 5 Regressions ( $\pm 95 \%$ confidence limits of means) of the proportion of dark shells on shore habitat PC2 (above) and mean standardized shell height (below). The separate regression lines for each island group do not differ significantly.

the substantial independence of geographic variation of these two traits, the large variation of morph frequencies and the repeatable association of colour, especially, with habitat. Taken together, these findings provide evidence of the genetic complexity of local adaptation in $B$. vittatum, and highlight the general importance of considering different types of traits when examining genetic structure.

\section{Association of colour and spotting}

The strong association of dark shells with spotted shells has important implications for the interpretation of geographic patterns of variation. The first implication is that the difference between pale shells and dark shells can be confidently interpreted as a genetic polymorphism. Although colour polymorphism is widespread in littorines, direct evidence for its genetic basis is rare (Reid, 1996), but is available for Littorina saxatilis (Ekendahl and Johannesson, 1997). In the case of $B$. vittatum, mating experiments confirmed the inheritance of the presence or absence of spots, consistent with a single locus, at which the spotted condition is dominant (Parsons, 1997). Thus, the strong association of colour and spotting is good evidence that the difference in colour is also heritable. The discrete colour morphs, combined with the association with spotting, also suggest that the inheritance is probably simple.

The strong association of colour and spotting within populations suggests either linkage of loci affecting the two traits, or epistasis, whereby spotting is more likely to develop in genetically dark shells. The fact that all four combinations of the two traits are found favours the simpler explanation of linked loci, in which case the 
question is whether the implied linkage disequilibrium is adaptive or is simply an historical artefact. The consistency of the association among these highly isolated local populations favours an adaptive interpretation. Although this interpretation is supported by the independent association of colour and spotting with habitat, discussed below, confident interpretation will require a more detailed understanding of the inheritance of these traits.

\section{Associations with habitat}

The contrasts of amounts and patterns of variation of colour, spotting and relative shell height with those of the allozymes are evidence for local adaptation of the shell traits. The substantially higher level of subdivision for the shell traits is typical of morphological traits (Spitze, 1993; Steinger et al., 2002; Storz, 2002; Luttikhuizen et al., 2003; Volis et al., 2005; Schiffer et al., 2007). The distribution of the variation is further evidence that the phenotypic variation is adaptive. Although the allozymes indicate clearly that the gaps between the island groups have caused substantial genetic isolation (Johnson and Black, 1996), that isolation has not affected the shell traits. Instead, the shells vary within island groups. The association of the proportions of colour morphs with shore type is strong evidence of local adaptation. The relationship is significant and indistinguishable among the three island groups. Independent of its association with colour, shell spotting shows a weaker association with habitat, but still with repeatability.

Studies of shell colour in intertidal gastropods have explained associations with habitat in terms of the two obvious selective mechanisms: (1) crypsis favoured by selective predation (Reid, 1987; Byers, 1995; Johannesson and Ekendahl, 2002; McKillup and McKillup, 2002; Parsonage and Hughes, 2002); and (2) thermal selection (Harris and Jones, 1995; Yeap et al., 2001; Parsonage and Hughes, 2002). Either mechanism could apply in the case of B. vittatum. Frequencies of dark shells are higher on vertical shores, which provide a greyish background. To the human eye, at least, the pale shells are conspicuous against this background, whereas the dark shells are relatively cryptic. At the other extreme of the shore types are gently sloping shores, with substrates of coral fragments and shells on sand. This habitat provides a paler but often more heterogeneous background, which could favour higher frequencies of pale shells, as well as greater heterogeneity. Thus, the distribution of shell morphs is consistent with selection favouring habitat matching. However, the contrasting habitats also differ in their thermal environments. Vertical shores are subject to less direct sunlight than are gently sloping shores, so the higher proportions of pale shells on the gently sloping shores fit a widespread pattern of pale shells being associated with sunnier habitats (Harris and Jones, 1995; Yeap et al., 2001; Parsonage and Hughes, 2002). Thus, although we do not have direct evidence about the mechanism of the local adaptation, the pattern of association of shell colour with habitat in B. vittatum is typical of that in many gastropods, and offers clear hypotheses for detailed testing.

Although predictions of a direct importance of colour were possible, based on findings in other snails, the same is not true for spotting of the shell. The spots are on the ventral surface, so they are seldom exposed either to visual predators or to sunlight. It is thus not surprising that spotting shows an intermediate level of subdivision to that of colour and the allozymes, and is only weakly associated with habitat. Nevertheless, the fact that there is some association of spotting with habitat, independent of its association with colour, indicates adaptive divergence of this trait. Although the mechanisms of local adaptation are not clear, spotting as well as colour appears to contribute to local adaptation in B. vittatum.

\section{Biologically significant environmental gradients}

Beyond providing good evidence of local adaptation of shell traits, the variation in B. vittatum is significant in its contrasting associations of the separate traits. Despite their association within sites, colour and spotting are only weakly associated among sites, indicating that their interaction imposes little constraint on divergence of the individual traits. In contrast, shell colour and shape are independent traits, not associated within populations, but they are well correlated in their variations among populations. The association is repeatable between the three island groups, so it is not simply an historical artefact, but instead indicates parallel contributions to local adaptation.

The parallel variation of colour and shape indicates a common, biologically significant axis. The extremes of that environmental gradient are obvious: the gently sloping shores and the vertical rock faces provide different substrates, backgrounds, exposure to the sun, exposure to waves and so on. Indeed, although shell colour and shape vary together along this gradient, the underlying mechanisms are undoubtedly different. Selection on colour is likely to be based on its appearance and thermal effects, whereas shell shape is more likely to be important for physical reasons; snails with tall shells are at a disadvantage on the vertical shores, because they cannot take refuge in crevices, so they are easily washed off the shore (Johnson and Black, 2000). Thus, the analysis of shell variation has revealed complex local adaptation, involving independent traits, and thereby highlighting the biological importance of this environmental gradient.

In addition to its importance for understanding processes of local adaptation, the distribution of adaptive genetic diversity is fundamental to issues of setting priorities in conservation biology. Genetic diversity is usually measured with molecular genetic markers, which may have little relationship to biologically significant variation. In $B$. vittatum, variation at 14 allozyme loci was sufficient to reveal detailed patterns of genetic connectivity of populations throughout the Abrolhos Islands, providing a good understanding of the overall structure of these populations (Johnson and Black 1995, 1996, 1998a, 2006). However, allozymes are not associated with any of the characteristics of the habitat that we have examined, either individually or as multivariate axes. That lack of association includes the biologically important gradient revealed by the common associations of shell colour, shape and to some degree spotting. This example highlights the importance of evaluating genetic differences between populations, using biologically significant traits. 
Genomic scans, using molecular markers such as AFLPs, are providing one means of finding loci that are candidates for local adaptation. Our findings for $B$. vittatum support the rationale for that approach, as the shell traits fit the predicted pattern of greater variation among populations than shown by presumably neutral markers. Thus, even in a species with a relatively high level of genetic subdivision, the greater variance of traits involved in local adaptation is evident.

Beyond the importance of the individual traits, however, the fact that both colour and shape vary along the same environmental gradient indicates the importance of that gradient, involving complex changes of independent traits. This is consistent with the results for another littorine, L. saxatilis, in which 15 of 306 AFLP loci examined showed distinctly large $F_{\mathrm{ST}}$, and all 15 were associated with the same habitat gradient as shell morphology (Wilding et al., 2001; Grahame et al., 2006). It seems unlikely that all these loci are directly associated with the shell dimorphism in L. saxatilis, so it may well be that there is a complex of independent adaptations to this significant intertidal gradient. The results from both $B$. vittatum and $L$. saxatilis highlight the potential usefulness of identifying not just individual traits that are adaptively important, but also significant environmental gradients. In their proposal to use genomic scans to identify markers associated with local adaptation, as a basis for assessing conservation priorities, Bonin et al. (2007) also found greatest divergence associated with apparently important environmental gradients. Thus, although the emphasis has been on the identification of adaptively important traits, an important question for further work is how widely are independent traits adapted to the same environmental gradients. Identification of contrasting habitats associated with complex adaptations will be important for understanding evolutionary processes and for establishing priorities in conservation to preserve those processes.

\section{Acknowledgements}

Darcy Hebbert, Michelle Stuckey and Caroline Fuery scored the shells, Kim Brooks, Karen Parsons, Jane Prince and Michelle Stuckey helped with the collections, and John Clarke, skipper of Tako, provided transport throughout the Houtman Abrolhos. Oliver Berry, Terrie Finston, Jason Kennington, Tom Schmidt and Eleanor O'Brien commented on the paper. The Western Australian Fisheries Department allowed us to use their field camps. The Australian Research Council funded the research.

\section{References}

Avise JC (2004). Molecular Markers, Natural History and Evolution, 2nd edn. Chapman and Hall: New York.

Beaumont MA (2005). Adaptation and speciation: what can $F_{\mathrm{ST}}$ tell us? Trends Ecol Evol 20: 435-440.

Bonin A, Nicole F, Pompanon F, Miaud C, Taberlet P (2007). Population adaptive index: a new method to help measure intraspecific genetic diversity and prioritise populations for conservation. Conservat Biol 21: 697-708.

Byers BA (1995). Shell color polymorphism associated with substrate color in the intertidal snail Littorina saxatilis Olivi (Prosobranchia, Littorinidae). Biol J Linnean Soc 40: 3-10.
Ekendahl A, Johannesson K (1997). Shell colour variation in Littorina saxatilis Olivi (Prosobranchia: Littorinidae): a multifactor approach. Biol J Linnean Soc 62: 401-419.

Gockel J, Kennington WJ, Hoffmann A, Goldstein DB, Partridge L (2001). Nonclinality of molecular variation implicates selection in maintaining a morphological cline of Drosophila melanogaster. Genetics 158: 319-323.

Grahame JW, Wilding CS, Butlin RK (2006). Adaptation to a steep environmental gradient and an associated barrier to gene exchange in Littorina saxatilis. Evolution 60: 268-278.

Harris DJ, Jones JS (1995). Habitat selection and thermal ecology in Nucella lapillus (L) (the dogwhelk). Heredity 74: 311-314.

Johannesson K, Ekendahl A (2002). Selective predation favouring cryptic individuals of marine snails (Littorina). Biol J Linnean Soc 76: 137-144.

Johnson MS, Black R (1995). Neighbourhood size and the importance of barriers to gene flow in an intertidal snail. Heredity 75: 142-154.

Johnson MS, Black R (1996). Geographic cohesiveness versus associations with habitat: genetic subdivision of Bembicium vittatum Philippi (Gastropoda: Littorinidae) in the Houtman Abrolhos Islands. Biol J Linnean Soc 58: 57-74.

Johnson MS, Black R (1997). Distributions of high-intertidal gastropods in the Houtman Abrolhos Islands. In: Wells FE (ed). The Marine Flora and Fauna of the Houtman Abrolhos Islands, Western Australia. Western Australian Museum: Perth. pp 101-112.

Johnson MS, Black R (1998a). Increased genetic divergence and reduced genetic variation in populations of the snail Bembicium vittatum in isolated tidal ponds. Heredity $\mathbf{8 0}$ 163-172.

Johnson MS, Black R (1998b). Effects of habitat on growth and shape of contrasting phenotypes of Bembicium vittatum Philippi in the Houtman Abrolhos Islands, Western Australia. Hydrobiologia 378: 95-103.

Johnson MS, Black R (2000). Associations with habitat versus geographic cohesiveness: size and shape of Bembicium vittatum Philippi (Gastropoda: Littorinidae) in the Houtman Abrolhos Islands. Biol J Linnean Soc 71: 563-580.

Johnson MS, Black R (2006). Islands increase genetic subdivision and disrupt patterns of connectivity of intertidal snails in a complex archipelago. Evolution 60: 2498-2506.

Lewontin RC, Krakauer J (1973). Distribution of gene frequency as a test of the selective neutrality of polymorphisms. Genetics 80: 175-195.

Luttikhuizen PC, Drent J, van Delden W, Piersma T (2003). Spatially structured genetic variation in a broadcast spawning bivalve: quantitative vs molecular traits. J Evol Biol 16: 260-272.

Lynch M (1996). A quantitative-genetic perspective on conservation issues. In: Avise JC, Hamrick JL (eds). Conservation Genetics: Case Histories From Nature. Chapman \& Hall: New York. pp 471-501.

McKay JK, Latta RG (2002). Adaptive population divergence: markers, QTL and traits. Trends Ecol Evol 17: 205-291.

McKillup SC, McKillup RV (2002). Flies that attack polymorphic snails on coloured backgrounds: selection for crypsis by a sarcophagid parasitoid of Littoraria filosa. Biol J Linnean Soc 77: 367-377.

McQuaid CD (1996). Biology of the gastropod family Littorinidae. 1. Evolutionary aspects. Oceanog Mar Biol 34: 233-262.

Nei M (1973). Analysis of gene diversity in subdivided populations. Proc Natl Acad Sci 70: 3321-3323.

Parsonage S, Hughes J (2002). Natural selection and the distribution of shell colour morphs in three species of Littoraria (Gastropoda: Littorinidae) in Moreton Bay, Queensland. Biol J Linnean Soc 75: 219-232.

Parsons KE (1997). Contrasting patterns of heritable geographic variation in shell morphology and growth potential in the 
marine gastropod Bembicium vittatum: evidence from field experiments. Evolution 51: 784-796.

Pearman PB (2001). Conservation value of independently evolving traits: sacred cow or testable hypothesis? Conserv Biol 15: 780-783.

Quesada H, Posada D, Caballero A, Moran P, Rolán-Alvarez E (2007). Phylogenetic evidence for multiple sympatric ecological diversification in a marine snail. Evolution 61: 1600-1612.

Reed DH, Frankham R (2001). How closely correlated are molecular and quantitative measures of genetic variation? A meta-analysis. Evolution 55: 1095-1103.

Reid DG (1987). Natural selection for apostasy and crypsis acting on the shell color polymorphism of a mangrove snail, Littoraria filosa (Sowerby) (Gastropoda, Littorinidae). Biol J Linnean Soc 30: 1-24.

Reid DG (1996). Systematics and Evolution of Littorina. The Ray Society: London.

Schiffer M, Kennington WJ, Hoffman AA, Blacket MJ (2007). Lack of genetic structure among ecologically adapted populations of an Australian rainforest Drosophila species as indicated by microsatellite markers and mitrochondrial DNA sequences. Mol Ecol 16: 1687-1700.

Schneider CJ, Smith TB, Larison B, Moritz C (1999). A test of alternative models of diversification in tropical rainforests: ecological gradients vs rainforest refugia. Proc Natl Acad Sci 96: 13869-13873.

Simon JL (1995). Resampling: The New Statistics. Resampling Stats Inc: Arlington.
Spitze K (1993). Population structure in Daphnia obtusa: quantitative genetic and allozymic variation. Genetics 135: 367-374.

Steinger T, Haldimann P, Leiss KA, Muller-Scharer H (2002). Does natural selection promote population divergence? A comparative analysis of population structure using amplified fragment length polymorphism markers and quantitative traits. Mol Ecol 11: 2583-2590.

Storz JF (2002). Contrasting patterns of divergence in quantitative traits and neutral DNA markers: analysis of clinical variation. Mol Ecol 11: 2537-2551.

Storz JF (2005). Using genome scans of DNA polymorphisms to infer adaptive population divergence. Mol Ecol 14: 671-688.

van Tienderen PH, de Haas AA, van der Linden CG (2002). Biodiversity assessment using markers for ecologically important traits. Trends Ecol Evol 17: 577-582.

Vermeij GJ (1993). A Natural History of Shells. Princeton University Press: Princeton.

Volis S, Yakubov B, Shulgina I, Ward D, Mendlinger S (2005). Distinguishing adaptive from nonadaptive genetic differentiation: comparison of $Q_{\mathrm{ST}}$ and $F_{\mathrm{ST}}$ at two spatial scales. Heredity 95: 466-475.

Wilding CS, Butlin RK, Graham J (2001). Differential gene exchange between parapatric morphs of Littorina saxatilis detected using AFLP markers. J Evol Biol 14: 611-619.

Yeap KL, Black R, Johnson MS (2001). The complexity of phenotypic plasticity in the intertidal snail Nodilittorina australis. Biol J Linnean Soc 72: 63-76. 